\title{
Bulk Fill Kompozit Rezinlerin İki Farklı Tabaka Kalınlığında ve Işık Gücünde Polimerize Edilmesinin Kompozitin Basma Dayanımına Etkisi
}

\author{
The Effect of Polymerizing Bulk Fill Composite Resins at Different Layer Thicknesses and Two \\ Different Light Power on the Compressive Strength of the Composite
}

\author{
Sevde Gül BATMAZ ${ }^{\mathbb{D}}$, Ayşe DÜNDAR ${ }^{\mathbb{D}}$, Çağatay BARUTÇUGIL ${ }^{\circledR}$, Berkan YILDIZ ${ }^{\mathbb{D}}$
}

Öz

Amaç: Bu çalışmanın amacı, iki farklı tabaka kalınlı̆̆ında ve iki farklı 1şık gücünde polimerize edilen üç bulk fill kompozit rezinin basma dayanımının değerlendirilmesidir.

Gereç ve Yöntem: Bu çalışmada, üç farklı bulk fill kompozit rezin (SonicFill 2, Kerr Dental, Orange, CA, ABD - Filtek Bulkfill 3M, ESPE, St. Paul, MN, ABD - EverX Posterior, GC, Leuven, Belçika) kullanıld. Her grupta 10 numune olacak şekilde $5 \mathrm{~mm}$ çapında, 4 ve $6 \mathrm{~mm}$ kalınlıklarında ve tek tabaka olarak iki farklı 1şık güç modunda ( $\left.1000 \mathrm{~mW} / \mathrm{cm}^{2}, 3 \mathrm{sn}-3200 \mathrm{~mW} / \mathrm{cm}^{2}, 20 \mathrm{sn}\right)$ ayrı ayrı polimerize edilerek 120 numune hazırlandı. Toplamda 12 grup oluşturuldu. Hazırlanan örneklerin alt ve üst yüzeylerini birbirine paralel hale getirilmek için Sof-Lex (3M ESPE, St Paul, MN, ABD) cila diskleri kullanıldı. Kalından inceye doğru sırası ile her bir örnek kuru ve tek yönlü olarak mikromotor ile düşük devirde (15000 rpm) cilalandı Teflon kalıplardan çıkarılan örneklerin kalınlıklarının standardizasyonunu sağlamak için örnekler dijital kumpas (Mitutoyo Digimatic) kullanılarak ölçüldü. Ardından örnekler Universal test cihazı (Zwick/Roell Z250, Almanya) kullanılarak, $1 \mathrm{~cm} / \mathrm{dk}$ çapraz kafa hızında basma dayanımı testine tabi tutuldu. Verilerin analizi için tek yönlü varyans analizi ve Tukey HSD testleri kullanıldı.

Bulgular: EverX Posterior kompozit rezinin $6 \mathrm{~mm}$ kalınlığındaki örneklerinde 1 şık şiddeti arttırıldığında basma dayanımı değerleri istatistiksel olarak anlamlı bir şekilde

Sevde Gül Batmaz (四)

Adana Fatma Kemal Timuçin Ağız ve Diş Sağllğ Merkezi, Adnan Kahveci, Bulvarı Iller Bankası Karşısı No:24, 01170 Çukurova, Adana, Türkiye. e-mail:sevde_isik@windowslive.com

Ayşe Dündar

Akdeniz Üniversitesi Diş Hekimliği Fakültesi Restoratif Diş Tedavisi ABD, Pınarbaşı Mahallesi Dumlupınar Bulvarı,Antalya, Türkiye

Çağatay Barutçugil

Akdeniz Üniversitesi Diş Hekimliği Fakültesi Restoratif Diş Tedavisi ABD, Pınarbaşı Mahallesi Dumlupınar Bulvarı, Konyaaltı, Antalya, Türkiye

Berkan Yildiz

Akdeniz Üniversitesi Diş Hekimliği Fakültesi Restoratif Diş Tedavisi ABD, Pınarbaşı Mahallesi Dumlupınar Bulvarı,Konyaaltı, Antalya, Türkiye

Submitted / Gönderilme: 19.12.2020

Accepted/Kabul: 03.06.2021 azalmıştır $(\mathrm{p}<0,05)$. Tabaka kalınlığının artması EverX Posterior kompozit rezin dışında basma dayanımını istatistiksel olarak anlamlı şekilde etkilememiştir ( $p>0,05)$. Tüm gruplar arasında en yüksek değeri ekstra güç modunda polimerize edilen $4 \mathrm{~mm}$ kalınlıktaki EverX Posterior örnekleri, en düşük değeri ise ekstra güç modunda polimerize edilen $6 \mathrm{~mm}$ kalınlıktaki Sonic Fill örnekleri göstermiştir.

Sonuç: Standart güç modunda polimerize edilen örneklerde tabaka kalınlığı basma dayanımını değerlerini etkilemezken, ekstra güç modunda tabaka kalınlığı basma dayanımı değerlerini etkilemektedir.

Anahtar Kelimeler: Basma dayanımı, bulk fill kompozit rezinler, tabaka kalınlığ 1

\section{Abstract}

Objective: The aim of this study is to determine the compressive strength values of three bulk fill composite resins polymerized at two different layer thicknesses and two different light power.

Material and methods: In this study, three different bulk fill composite resins (SonicFill 2, Kerr Dental, Orange, CA, USA Filtek Bulkfill 3M, ESPE, St. Paul, MN, USA - EverX Posterior, GC, Leuven, Belgium) were used. A total of 10 samples in each group, $5 \mathrm{~mm}$ in diameter, 4 and $6 \mathrm{~mm}$ thick, and each thickness was polymerized separately in two different light power $(1000 \mathrm{~mW} /$ $\mathrm{cm}^{2}, 3 \mathrm{sec}-3200 \mathrm{~mW} / \mathrm{cm}^{2}, 20 \mathrm{sec}$ ) modes as single layer and 120 samples were prepared. A total of 12 groups were created. Sof-Lex (3M ESPE, St Paul, MN, USA) polish discs were used to make the upper and lower surfaces of the prepared samples parallel to each other. The samples were measured using digital calipers (Mitutoyo Digimatic) in order to standardize the thickness of the samples extracted from Teflon molds. One-way analysis of variance and Tukey HSD tests were used to analyze the data. Subsequently, the compressive strength test was performed on samples using a Universal test device (Zwick / Roell Z250, Germany) at $1 \mathrm{~mm} /$ min crosshead speed. One-way analysis of variance and Tukey HSD tests were used for data analysis.

Results: When the light intensity was increased in $6 \mathrm{~mm}$ thick samples of EverX Posterior composite resin, the compressive strength values decreased statistically significantly $(p>0.05)$. The increase in layer thickness did not significantly affect compression strength, except for EverX Posterior composite resin $(\mathrm{p}>0.05)$. 
Among all groups, the $4 \mathrm{~mm}$-thick EverX Posterior samples polymerized in the extra power mode had the highest value, while the lowest value was the $6 \mathrm{~mm}$-thick Sonic Fill samples polymerized in the extra power mode.

Conclusion: While the layer thickness does not affect the compressive strength values in the samples polymerized in the standard power mode, layer thickness in the extra power mode affects the compressive strength values.

Keywords: Compressive strength, bulk fill composite resins, layer thickness

\section{Giriş}

Günümüzde kompozit rezinler, kolay kullanımları, estetik özelliklerinin yeterli olması, mekanik özellikleri, diş yapısına bağlanmaları ve seramiklere kıyasla daha düşük maliyete sahip olmaları nedeniyle klinik uygulamalarda yaygın olarak kullanılmaktadır (Barutcigil, Barutcigil, Özarslan, Dündar, \& Yilmaz, 2018). Kompozit rezinler, diş hekimliği alanında kullanılmaya başladığı günden bugüne kadar, monomer kimyası, doldurucu teknolojisi ve yapısı, fizikokimyasal özellikleri sürekli olarak geliştirilmiştir (Ilie \& Hickel, 2011).

Üreticiler, rezin esaslı kompozitlerin posterior bölgede büyük tabakalar halinde yerleştirilmesini basitleştirmek ve hızlandırmak için tek tabaka halinde veya daha kalın tabakalar halinde yerleştirilebilen, bulk fill rezin kompozitleri üretmişlerdir. Nispeten daha kısa bir süre zarfında, bulk fill rezin kompozitlerin 4-10 mm arasında bir tabaka kalınlığında kullanılabileceği belirtilerek piyasaya sürülmüştür (Chesterman, Jowett, Gallacher, \& Nixon, 2017). Hekime uygulama ve kullanım kolaylığı sağlaması, tabakalar arasında boşluk kalma olasılığının az olması, yeterli radyoopasiteye sahip olması, yüzey özellikleri ve renk uyumunun klinik olarak kabul edilebilir seviyede olması ve çiğneme kuvvetlerine karşı aşınma direncinin iyi olması bulk fill kompozitlerin avantajları arasında yer almaktadır (Aydın, Karaoğlanoğlu, Oktay, Topçu, \& Demir). Günümüzde restoratif diş hekimliğinde tercih edilen başka bir yöntem de kompozit rezinlerin fiberle güçlendirilmesidir. Dentinin stres emici özelliklerini taklit etmek amacıyla 2013 yılında kısa fiberle güçlendirilmiş kompozit rezin (everX Posterior; GC, Tokyo, Japonya) piyasaya sürüldü. $\mathrm{Bu}$ kompozit rezinlerin yüksek gerilim taşıyan alanlarda, vital ve devital dişlerin restorasyonunda taban materyali olarak kullanılması amaçlanmıştır. Fiberle güçlendirilmiş kompozit rezinler, bir rezin matrisi, rastgele yönlendirilmiş E-cam fiberler ve inorganik partikül doldurucu maddelerinin kombinasyonundan oluşur (Garoushi, Gargoum, Vallittu, \& Lassila, 2018).
Genel olarak bulk fill kompozit rezinlerin kimyasal yapısı geleneksel kompozitlere benzerlik göstermektedir. Kompozit rezinlerin doldurucu tipi, bileşimi, boyutu ve ağırlık yüzdesi, polimerizasyon kalitesi ve derinliği, $1 s ̧ 1 \mathrm{k}$ kaynağı, ışık kaynağının özellikleri, matris oranı, doldurucu miktarı ve boyutu gibi çok sayıda faktör, bu materyallerin aşınma direnci, sertlik, basma ve eğilme dayanımı gibi fiziksel ve mekanik özellikleri etkilemektedir (Alkhudhairy \& Vohra, 2016; Vandewalker, Casey, Lincoln, \& Vandewalle, 2016).

Dental restorasyonlar, çiğneme sırasında basınç, çekme ve kesme kuvvetleri dâhil karmaşık yükleme senaryolarına maruz kalır. Bu kuvvetler arasında çekme kuvvetleri kritik olarak belirtilmiş olsa da basma kuvvetleri de endişe kaynağıdır (Cho, Kaneko, Donovan, \& White, 1999; Drummond, 2008; Sunnegårdh-Grönberg, Peutzfeldt, \& van Dijken, 2003).

Basma dayanımı, sıkıştırılmış test numunesinin kırılma noktasındaki basınç dayanımı olarak tanımlanır. Kuvvet, yalnızca atomik çekim veya itmenin bir ölçümü olarak değil, aynı zamanda sıkıştırılmış yapının atomlar arası kuvvetlerinin toplu bir ölçümü olarak da ifade edilir (K. Anusavice, 1996; K. J. Anusavice, Shen, \& Rawls, 2012). Çiğneme eylemi sırasında restorasyonlara iletilen kuvvetlerin onları kırabileceği veya dişin kırılmasına sebep olabileceği bilinmektedir. Çiğneme kuvvetlerinin çoğu sıkıştırıcı nitelikte olduğundan, basma dayanımı, çiğneme sürecinde özellikle önemli bir role sahiptir (Rueggeberg, Caughman, \& Curtis, 1994).

S1kıştırmaya karşı maksimum direnç, test numunesinin orijinal kesit alanı ve uygulanan maksimum kuvvet ile hesaplanır. Klinik olarak ilgili bir basma dayanımı değeri, mineralize dokuların basma dayanımı değerlerine bağlı olabilir. Minenin basma dayanımı $384 \mathrm{MPa}$, dentinin basma dayanımı ise $287 \mathrm{MPa}$ olarak ölçülmüştür (Tyler \& Poole, 1984). Posterior dişlerinin basma dayanımı $305 \mathrm{MPa}$ civarında iken, diğer dişler genellikle daha düşük basma dayanımına sahiptir. Posterior dişlerin basma dayanımı, bu bölgede kullanılan kompozit rezinlerin optimum mukavemetini seçmek için iyi bir mekanik standart sunabilir (Galvão et al., 2013; Willems, Lambrechts, Braem, \& Vanherle, 1993).

Restoratif materyallerin yorulma sınırını belirlemek dental restorasyonların uzun ömürlülüğü hakkında daha güvenilir bilgi sağlayabileceğinden dolayı önemlidir. $\mathrm{Bu}$ çalışmanın amacı, iki farklı tabaka kalınlığında ve iki farklı 1şık gücünde polimerize edilen üç farklı bulk fill kompozit 
rezinin basma dayanımı değerlerinin belirlenmesidir. Test edilen sifir hipotezler:

(I) Kompozitlerin tabaka kalınlı̆̆ı, kompozit rezinlerin basma dayanımı değerlerini etkilemez.

(II) Uygulanan 1şık şiddeti, kompozit rezinlerin basma dayanımı değerlerini etkilemez.

\section{Gereç ve Yöntem}

\section{Çalışmada kullanılan kompozit rezinler}

Bu çalışmada üç farklı bulk fill kompozit kullanıldı: EverX Posterior, Sonic Fill 2 ve Filtek Bulk Fill. Çalışmada kullanılan kompozit rezinler ve içerikleri Tablo 1'de gösterilmektedir.

\section{Örnek hazırlanması ve deneyin yapılması}

Örneklerin hazırlanması için $5 \mathrm{~mm}$ çapında, 4 ve 6 mm kalınlığında, 2 farklı teflon kalıp kullanıldı. Örnek hazırlanmadan önce teflon kalıbın alt yüzeyine şeffaf bant yerleştirildi. Bulk fill kompozitler, siman fulvarı yardımıyla iyice kondense edilerek tek tabaka olarak teflon kalıba yerleştirildi ve yüzeyi düzeltildi. Daha sonra, kalıbın üst yüzeyinden Valo 1şık cihazı (Ultradent, South Jordan, UT, USA) kullanılarak ışıklandı. Işık cihazı, gruplardan bir tanesinde ekstra güç modunda (3200 mW/cm2-3 sn), diğerinde ise standart güç modunda $(1000 \mathrm{~mW} / \mathrm{cm} 2-$ 20 sn) kullanıldı. Üç farklı bulk fill rezin kompozit, iki farklı 1şık gücü ve iki farklı tabaka kalınlığında, her bir grup için 10 örnek olmak üzere, toplamda 120 adet örnek hazırlanarak gruplandırıldı (Tablo 2). Örneklerin alt ve yüzeylerinin birbirine paralel hale getirilmesi için SofLex (3M ESPE Dental Products, St Paul, MN, USA) cila diskleri kullanılarak kalından inceye doğru sırası ile her bir örnek kuru ve tek yönlü olarak mikromotor ile 15000 rpm devirde cilalanmıştır. Örneklerin kalınlıklarını ölçmek için dijital kumpas (Mitutoyo Digimatic) kullanılmıştır. Basma dayanımı testi, Universal test cihazı (Zwick/Roell Z250, Almanya) kullanılarak, $1 \mathrm{~mm} / \mathrm{dk}$ çapraz kafa hızında yapıldı. Test uygulanırken ön yük 0,5 Newton eksenel olarak uygulandi.

\section{İstatistiksel Analiz}

Çalışmada elde edilen veriler, SPSS 23 istatistiksel yazılımı ( SPSS Inc, Chicago, IL) ile analiz edildi. Gruplar arası analizlerde tek yönlü varyans analizi ve Tukey HSD testleri kullanıldı. Tüm istatistiksel analizlerde, anlamlılık düzeyi $\% 95$ olarak belirlendi.

\section{Bulgular}

Farklı tabaka kalınlıkları ve farklı 1şık güçlerinde polimerizasyonun, materyalin basınç kuvvetlerine direncinin karşılaştırılmalı olarak değerlendirildiği bu çalışmada, gruplara ait ortalama ve standart sapma değerleri Megapaskal (MPa) cinsinden Tablo 3'te gösterilmektedir. Basma dayanımı bulguları incelendiğinde tüm gruplar arasında en yüksek değeri ekstra güç modunda polimerize edilen $4 \mathrm{~mm}$ kalınlığındaki EverX Posterior kompozit rezin örnekleri, en düşük değeri ise ekstra güç modunda polimerize edilen $6 \mathrm{~mm}$ kalınlığındaki Sonic Fill kompozit rezin örnekleri göstermiştir. Ekstra güç modunda polimerize edilen diğer gruplar arasında istatistiksel olarak anlamlı fark bulunamamıştır ( $\mathrm{p}>0,05)$ (Tablo 3).

Tablo 1. Çalışmada kullanılan bulk fill kompozit rezinler ve içerikleri

\begin{tabular}{|c|c|c|c|c|}
\hline Bulk fill kompozit & İçerik & $\begin{array}{c}\text { Doldurucu oranı } \\
\text { (Ăgrılı/Hacim) }\end{array}$ & LOT numarası & Üreitici Firma \\
\hline EverX Posterior & $\begin{array}{c}\text { Bis-GMA, TEGDMA, } \\
\text { silikondioksit, baryum cam, } \\
\text { cam fiber, polimetilmetakrilat, } \\
\text { fotobaşlatıc1 }\end{array}$ & $\% 74,2 / \% 53,6$ & 1904012 & GC, Leuven, Belçika \\
\hline Filtek Bulk Fill & $\begin{array}{c}\text { Bis-GMA, UDMA, Bis-EMA } \\
\text { ytterbiyum triflorid,zirkon } \\
\text { silika }\end{array}$ & $\% 76,5 / \% 58,4$ & NA33172 & $3 \mathrm{M}$ Espe, St.Paul, ABD \\
\hline Sonic Fill 2 & $\begin{array}{c}\text { Bis-GMA, TEGDMA, } \\
\text { EBPADMA cam, oksit, silikon } \\
\text { dioksit }\end{array}$ & $\% 81,3 / \% 66$ & 6383679 & Kerr, Orange, CA, ABD \\
\hline
\end{tabular}

(Bis-GMA: Bisfenol-A-diglisidil-dimetakrilat, UDMA:Üretan Dimetakrilat, Bis-EMA:Bis Fenol A Etoksi Dimetakrilat, TEGDMA:Trietilen Glikol Dimetakrilat, EBPADMA: Etoksile bisfenol dimetakrilat) 
Standart güç modunda polimerize edilen örneklerin basma dayanımı değerleri incelendiğinde EverX Posterior ve Filtek Bulk Fill kompozit rezinlerin her iki kalınlıkta hazırlanan örnekleri arasında istatistiksel olarak anlamlı bir fark görülmemiştir (p>0,05) (Şekil 1) (Tablo 3).

Standart güç modunda Sonic Fill kompozit rezin örnekleri en düşük değerleri gösterirken, aynı kompozitin iki farklı kalınlıkta hazırlanan örnekleri arasında istatistiksel olarak anlamlı bir fark bulunamamıştır (p>0,05) (Şekil 1) (Tablo 3).
Işık şiddetinin basma dayanımına etkisi incelendiğinde Sonic Fill ve Filtek Bulk Fill kompozit rezinlerde 1şıı şiddetinin basma dayanımına istatistiksel olarak anlamlı bir etkisi gözlenmemiştir (p>0,05). Sadece EverX Posterior kompozit rezinin $6 \mathrm{~mm}$ kalınlığındaki örneklerinde ışık şiddeti arttırıldığında basma dayanımı değerleri istatistiksel olarak anlamlı bir şekilde azalmıştır $(\mathrm{p}<0,05)$. Tabaka kalınlığının artması EverX Posterior kompozit rezin dışında basma dayanımını istatistiksel olarak etkilememiştir $(\mathrm{p}<0,05)$ (Şekil 1).

Tablo 2. Farklı kalınlıklarda ve farklı 1şık güçlerinde hazırlanan kompozitlere ait deney grupları

\begin{tabular}{|c|c|c|c|c|c|}
\hline \multirow{2}{*}{\multicolumn{2}{|c|}{$\begin{array}{l}\text { Numune Kalınlığı (mm) } \\
\text { Işık Gücü }\left(\mathrm{mW} / \mathrm{cm}^{2}\right)\end{array}$}} & \multicolumn{2}{|l|}{$4 \mathrm{~mm}$} & \multicolumn{2}{|l|}{$6 \mathrm{~mm}$} \\
\hline & & $1000 \mathrm{~mW} / \mathrm{cm}^{2}$ & $3200 \mathrm{~mW} / \mathrm{cm}^{2}$ & $1000 \mathrm{~mW} / \mathrm{cm}^{2}$ & $3200 \mathrm{~mW} / \mathrm{cm}^{2}$ \\
\hline \multirow{3}{*}{ 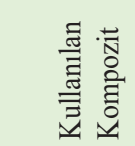 } & EverX Posterior & $\mathrm{n}=10$ & $\mathrm{n}=10$ & $n=10$ & $n=10$ \\
\hline & Filtek Bulk fill & $\mathrm{n}=10$ & $\mathrm{n}=10$ & $\mathrm{n}=10$ & $\mathrm{n}=10$ \\
\hline & Sonic Fill 2 & $n=10$ & $n=10$ & $n=10$ & $n=10$ \\
\hline
\end{tabular}

Tablo 3. Kompozit rezinlerin farklı tabaka kalınlıklarında ve farklı 1şık güçlerinde hazırlanan örneklerinin basma dayanımı değerleri

\begin{tabular}{|c|c|c|c|}
\hline \multirow{2}{*}{ Materyal } & \multirow{2}{*}{ Kalınlık } & \multicolumn{2}{|l|}{ Polimerizasyon yöntemi } \\
\hline & & Standart gücü modu $\left(1000 \mathrm{~mW} / \mathrm{cm}^{2}, 20 \mathrm{sn}\right)$ & Ekstra güç modu $\left(3200 \mathrm{~mW} / \mathrm{cm}^{2}, 3 \mathrm{sn}\right)$ \\
\hline \multirow{2}{*}{ Sonic Fill } & $6 \mathrm{~mm}$ & $223,59 \pm 16,94^{b}$ & $220,01 \pm 30,93^{\mathrm{A}}$ \\
\hline & $4 \mathrm{~mm}$ & $237,28 \pm 41,52^{b}$ & $235,98 \pm 21,77^{\mathrm{AB}}$ \\
\hline \multirow{2}{*}{ Ever X } & $6 \mathrm{~mm}$ & $324,23 \pm 19,06^{\text {a }}$ & $257,04 \pm 36,96^{\mathrm{AB}}$ \\
\hline & $4 \mathrm{~mm}$ & $326,22 \pm 42,23^{\text {a }}$ & $338,50 \pm 34,76^{\mathrm{C}}$ \\
\hline \multirow{2}{*}{ Filtek Bulk fill } & $6 \mathrm{~mm}$ & $289,83 \pm 20,48^{\text {a }}$ & $259,84 \pm 46,09^{\mathrm{AB}}$ \\
\hline & $4 \mathrm{~mm}$ & $316,12 \pm 25,61^{\text {a }}$ & $279,23 \pm 56,86^{\mathrm{B}}$ \\
\hline
\end{tabular}

Aynı sütundaki farklı üst karakterler istatististiksel olarak farklı grupları göstermektedir $(\mathrm{p}<0,05)$.

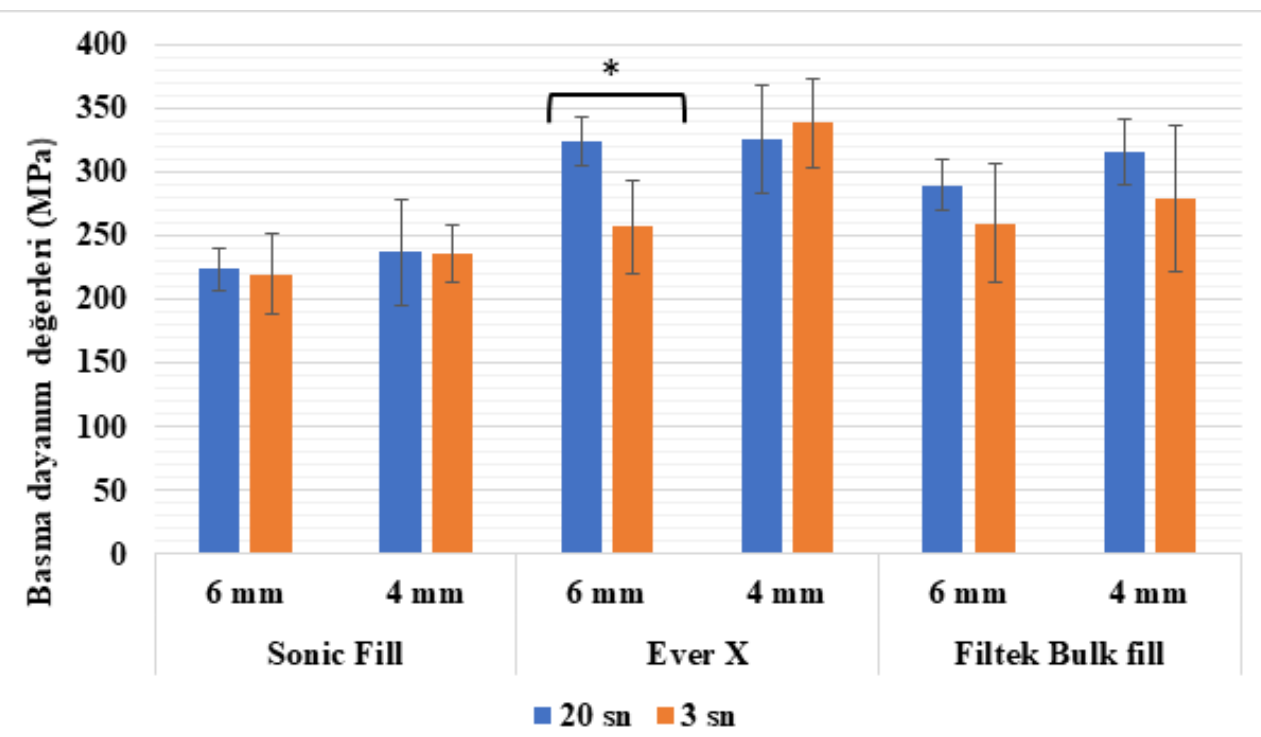

Şekil 1: Kompozit rezinlerin farklı tabaka kalınlıklarında ve farklı 1şık güçlerinde hazırlanan örneklerinin basma dayanımı değerleri (MPa) Gruplar arası istatistiksel farklılık * işaretiyle gösterilmiştir $(\mathrm{p}<0,05)$. 


\section{Tartışma}

Çiğneme işlevi ve parafonksiyondan kaynaklanan kuvvetler, restoratif materyallerin başarısını doğrudan etkiler. $\mathrm{Bu}$ sebeple restoratif materyallerin mekanik özellikleri, uzun dönemdeki başarının değerlendirilmesinde oldukça önem kazanmaktadır. Kompozit rezinlerin mekanik özellikleri ve klinik performansı farklı değişkenlerden etkilenir (Passos et al., 2013). Bu değişkenlerden birisi de kompozitlerin polimerizasyonudur (Ishikiriama, Valeretto, Franco, \& Mondelli, 2012). Kompozit rezinler etkili bir şekilde polimerize edilmezlerse, kürlenmemiş veya kısmen kürlenmiş kompozit rezin alanları tabanda veya tabakalar arasında kalarak dayanımda azalmaya sebep olabilir (Chesterman et al., 2017). Kompozit rezinlerde tabaka kalınlığı arttıkça 1şık geçirgenliği azalır. Bulk fill kompozitlerde ise artmış translusens ve polimerizasyon modülatörlerinin varlığıyla bu durumun üstesinden gelindiği iddia edilmektedir (Nascimento et al., 2018). Çalışmamızda geleneksel kompozitlere kıyasla nispeten daha kalın tabakalar kullanılmış ve tabaka kalınlığı arttıkça sadece ekstra güç modunda polimerize edilen EverX Posterior örneklerinde basma dayanımı değerleri azalmıştır. $\mathrm{Bu}$ sebeple ilk sıfır hipotezimiz kısmen reddedilmiştir.

Son yıllarda diş hekimliği alanında meydana gelen gelişmelerden birisi de uygulama zamanını azaltmada etkili olan; 1şık yoğunluğu arttırılmış üçüncü nesil LED 1şık cihazlarının kullanıma sunulmasıdır (Lee, Kim, Kang, \& Chang, 2016). Bu cihazlar çoklu dalga boyuna sahip olup kamferokinon dışındaki polimerizasyon başlatıcılarını da polimerize edilebilmektedir (Price, Fahey, \& Felix, 2010). Üçüncü nesil LED ışık cihazlarının dental restoratif materyallerin tamamında yeterli polimerizasyonu sağladığ1 bildirilmiştir (Lee et al., 2016; Spranley, Winkler, Dagate, Oncale, \& Strother, 2012). Çalışmamızda da üçüncü nesil LED 1şık cihazı (Valo Ultradent, South Jordan, UT, USA) iki farklı 1şık gücünde kullanılmıştır. Işık şiddeti arttıkça EverX Posterior bulk fill kompozitin $6 \mathrm{~mm}$ tabaka kalınlığında hazırlanan örneklerinde basma dayanımı istatistiksel olarak anlamlı derecede azalmıştır $(\mathrm{p}<0,05)$. Diğer gruplarda basma dayanımındaki azalmalar istatistiksel olarak anlamlı değildir $(\mathrm{p}>0.05)$. Bu durum, EverX Posterior içerisinde bulunan fiberlerin, tabaka kalınlığ 1 arttıkça 1 şık geçirgenliğini azaltmasıyla ve dolayısıyla derecesinin azalmış olma ihtimaliyle açıklanabilir. Dönüşüm derecesi ise dayanım, sertlik, çözünürlük, renk değişiklikleri ve biyouyumluluk gibi fiziksel ve mekanik özellikler ile doğrudan ilgilidir (Jain, Mehta, Meena, \& Gupta, 2018). Işı1k şiddetindeki değişmeler bazı kompozit gruplarında basma dayanımını etkilediğinden dolayı ikinci sıfır hipotezimiz kısmen reddedilmiştir.

Kompozitlerde kimyasal bileşimin mekanik özellikleri etkileyebileceği literatürde çeşitli çalışmalarda gösterilmiştir (Koupis, Vercruysse, Marks, Martens, \& Verbeeck, 2004; Soh, Yap, \& Siow, 2003; Tolosa, Paulillo, Giannini, Santos, \& Dias, 2005). Kompozitlerin mekanik özelliklerinin doldurucu içerikleri, doldurucu türü ve boyutu ile ilgili olduğu bilinmektedir (Kim, Ong, \& Okuno, 2002; Li, Swartz, Phillips, Moore, \& Roberts, 1985). Doldurucu içerikleri incelendiğinde doldurucu miktarı en yüksekten en düşüğe şu şekilde sıralanmaktadır: Sonic Fill 2 (ağırlıkça $\% 81,3$ / hacimce \%66), Filtek Bulk Fill (ağırlıkça \%76,5 / hacimce \%58,4) ve EverX Posterior (ağırlıkça \%74,2 / hacimce \%53,6). Fiberlerin esneklik, sertlik, basınca karşı dayanıklılık, düşük özgül ağırlık, translüsentlik, korozyona direnç ve adeziv teknik ile bağlanabilmeleri gibi özellikleri kompozit rezinlerin güçlendirilmesinde ve mekanik özelliklerinin iyileştirilmesinde de tercih edilmelerini sağlamıştır (Freilich, Karmaker, Burstone, \& Goldberg, 1998; van Dijken \& Sunnegårdh-Grönberg, 2006; H. H. Xu, Quinn, Smith, Giuseppetti, \& Eichmiller, 2003). Fiber ağların, uygulandığı kompozit rezinin bir bütün hâlinde kalmasını sağladığı ve bu şekilde dayanım değerlerini arttırdığını bildiren çeşitli çalışmalar da mevcuttur (Maruo, Nishigawa, Irie, Yoshihara, \& Minagi, 2015; Monaco, Bortolotto Ibarra, Arena, \& Krejci, 2015). EverX Posterior'un doldurucu oranı en düşük materyal olmasına rağmen en yüksek basma dayanımı değerlerini göstermesinin, kompozit yapının maruz kaldığı kuvveti, fiberleri çevreleyen rezin matriks tarafından fiberlere etkin olarak iletilmesiyle (H. Xu, 2000) ilgili olabileceğini düşünmekteyiz.

Klinik olarak ilgili basma dayanımı değeri, doğal mineralize dokuların basınç dayanımı değerlerine bağlı olabilir. Molar dişlerin kırılma dayanımı $305 \mathrm{MPa}$ civarında iken, diğer dişler genellikle daha düşük kırılma dayanımına sahiptir (Galvão et al., 2013). Bu değer, posterior dişlerde kullanılan kompozit rezinlerin optimum dayanımını seçmek için iyi bir mekanik standart sunabilir. Bu standarta göre Sonic Fill 2 kompozit rezin bu değerin altında kalmaktadır. EverX Posterior kompozit rezin ise $6 \mathrm{~mm}$ tabaka kalınlığı ve yüksek 1şık gücü modunda polimerize edilen örnekler dışında bu standartı sağlamıştır. Filtek Bulk Fill kompozit rezinin ise $4 \mathrm{~mm}$ tabaka kalınlığında ve her iki sşık gücünde hazırlanan örnekleri bu standartı sağlamıştır. 
Atabek ve arkadaşlarının 2014 yılında yaptıkları çalışmada, düşük stresli akışkan bir rezin materyali (SDR), bir bulk fill kompozit rezin (Tetric Evo Ceram), evrensel bir posterior kompozit (GC G-aenial) ve Sonic fill sistemi karşılaştırılmıştır. Çalışma sonucunda, Sonic fill sistemi (316.15 MPa) diğer gruplardan anlamlı olarak daha yüksek basma dayanımı değeri göstermiştir. Çalışmamızda ise en düşük basma dayanımı değerlerinden birini, Atabek ve arkadaşlarının hazırladıkları tabaka kalınlığında hazırlanan Sonic Fill 2 kompozit (223,59 MPa) rezin örnekleri göstermiştir (Didem \& Yalcin, 2014). Hazırlanan örneklerin çaplarındaki farklılıklar birim alana gelen kuvvetin değişmesine neden olmaktadır. Bu yüzden çalışmaların sayısal değerleri arasında farklılıklar oluşabilmektedir.

Galvao ve arkadaşları yaptıkları çalışmada, nanofil ve mikrohibrit kompozitlerin LED ışık cihazına takılan farklı ışık uçlarıyla (fiber optik ve polimer) polimerize edilmesinin kompozitin basma dayanımına etkisini araştırmışlardır (Galvão et al., 2013). Fiber optik uç kullanılan LED 1şık cihazında çıkan 1 şı şiddeti $653 \mathrm{~mW} / \mathrm{cm}^{2}$, polimer uç kullanılan cihazda ise $596 \mathrm{~mW} / \mathrm{cm}^{2}$ dir. Çalışma sonucunda fiber optik uç kullanılarak polimerize edilen örneklerin, polimer uç kullanılan örneklerden daha yüksek basma dayanımı değerleri gösterdiği bildirilmiştir. Çalışmamızda ise 1şı şiddetinin artması, sadece EverX Posterior kompozit rezinin $6 \mathrm{~mm}$ kalınlığa sahip örneklerin basma dayanım değerlerini istatistiksel olarak anlamlı şekilde azaltırken, $4 \mathrm{~mm}$ kalınlıktaki örneklerinde istatistiksel olmasa da ufak bir artışa neden olmuştur.

$\mathrm{Bu}$ çalışmanın bazı sınırlamaları mevcuttur. Çalışma in vitro şartlarda yapıldığı için ağız ortamını direkt taklit etmemektedir. Ağız içerisinde tükürük, kimyasal korozyon, termal değişiklikler ve yiyecekler gibi çeşitli faktörler de kompozit rezinlerin basma dayanımını etkileyecektir (Garoushi, Lassila, Tezvergil, \& Vallittu, 2007; Wang, D’Alpino, Lopes, \& Pereira, 2003). Ayrıca ağız içerisinde materyal sadece dikey yönde kuvvete maruz kalmamakta, farklı yönden gelen birçok kuvvete maruz kalmaktadır. Bu sebeple bu kuvvetlerin de dâhil edilebildiği bir çalışma, sonuçları etkileyebilir. Bir diğer faktör, örnek hazırlarken 1şık cihazı mümkün olan en yakın mesafeden ve örneklere dik olacak şekilde konumlandırılmışken, bunu ağız ortamında standardize etmek her zaman mümkün olmayabilir.

Farklı tabaka kalınlıklarında ve farklı ışık güçlerinde hazırlanan bulk fill kompozit rezinlerin basma dayanımının incelendiği bu in vitro çalışmada elde edilen değerler; bu materyallerin, termal, kimyasal ve fiziksel streslere maruz bırakılması sonrasında değişkenlik gösterebilmektedir. Bu sebeple, farklı etkenlerin yer aldığı uygulamaların in vitro ve in vivo koşullarda etkilerini değerlendirebilmek için bu yönde çalışmalar yapılması gerekmektedir.

\section{Sonuç}

$\mathrm{Bu}$ çalışmanın sınırlamaları dâhilinde, aşağıdaki sonuçlar elde edilmiştir:

Kompozit rezinler arasında en yüksek basma dayanımı değerlerini EverX Posterior kompozit rezin, en düşük basma dayanımı değerlerini ise Sonic Fill 2 kompozit rezin göstermiştir.

Işık şiddetinin artması sadece EverX Posterior kompozit rezinin $6 \mathrm{~mm}$ tabaka kalınlığındaki örneklerinde istatistiksel olarak anlamlı bir azalmaya sebep olurken, diğer gruplarda istatistiksel bir değişikliğe sebep olmamıştır.

Kompozitlerin tabaka kalınlığının artması EverX Posterior kompozitin yüksek 1şık gücünde polimerize edilen örnekleri dışında, basma dayanımı değerlerinde istatistiksel olarak anlamlı bir fark yaratmamıştır.

\section{Kaynaklar}

1. Alkhudhairy, F., \& Vohra, F. (2016). Compressive strength and the effect of duration after photo-activation among dualcure bulk fill composite core materials. PaK J Med Sci, 32(5), 1199.

2. Anusavice, K. (1996). Mechanical properties of dental materials. Phillip's science of dental materials, 457, 493.

3. Anusavice, K. J., Shen, C., \& Rawls, H. R. (2012). Phillips' science of dental materials: Elsevier Health Sciences.

4. Aydın, N., Karaoğlanoğlu, S., Oktay, E. A., Topçu, F. T., \& Demir, F. Diş hekimliğinde bulk fill kompozit rezinler. Selcuk Dental Journal, 6(2), 229-238.

5. Barutcigil, Ç., Barutcigil, K., Özarslan, M. M., Dündar, A., \& Yilmaz, B. (2018). Color of bulk-fill composite resin restorative materials. J. Esthet. Dent, 30(2), E3-E8.

6. Chesterman, J., Jowett, A., Gallacher, A., \& Nixon, P. (2017). Bulk-fill resin-based composite restorative materials: a review. Br. Dent. J., 222(5), 337-344.

7. Cho, G. C., Kaneko, L. M., Donovan, T. E., \& White, S. N. (1999). Diametral and compressive strength of dental core materials. J Prosthet Dent, 82(3), 272-276.

8. Didem, A., \& Yalcin, G. (2014). Comparative mechanical properties of bulk-fill resins. J. Compos. Mater., 2014.

9. Drummond, J. L. (2008). Degradation, fatigue, and failure of resin dental composite materials. J. Dent. Res., 87(8), 710719. 
10. Freilich, M. A., Karmaker, A. C., Burstone, C. J., \& Goldberg, A. J. (1998). Development and clinical applications of a light-polymerized fiber-reinforced composite. Prosthet Dent, 80(3), 311-318.

11. Galvão, M., Caldas, S. G. F. R., Calabrez-Filho, S., Campos, E. A. d., Bagnato, V. S., Rastelli, A. N. d. S., \& Andrade, M. F. (2013). Compressive strength of dental composites photoactivated with different light tips. Laser Phys, 23(4), 045604.

12. Garoushi, S., Gargoum, A., Vallittu, P. K., \& Lassila, L. (2018). Short fiber-reinforced composite restorations: a review of the current literature. J Investig Clin Dent, 9(3), e12330.

13. Garoushi, S., Lassila, L. V., Tezvergil, A., \& Vallittu, P. K. (2007). Static and fatigue compression test for particulate filler composite resin with fiber-reinforced composite substructure. Dent. Mater., 23(1), 17-23.

14. Ilie, N., \& Hickel, R. (2011). Investigations on a methacrylatebased flowable composite based on the SDR ${ }^{\mathrm{TM}}$ technology. Dent. Mater., 27(4), 348-355.

15. Ishikiriama, S. K., Valeretto, T. M., Franco, E. B., \& Mondelli, R. F. L. (2012). The influence of" C-factor" and light activation technique on polymerization contraction forces of resin composite. J. Appl. Oral Sci, 20(6), 603-606.

16. Jain, L., Mehta, D., Meena, N., \& Gupta, R. (2018). Influence of light energy density, composite type, composite thickness, and postcuring phase on degree of conversion of bulk-fill composites. Contemp. Clin. Dent, 9(Suppl 1), S147.

17. Kim, K.-H., Ong, J. L., \& Okuno, O. (2002). The effect of filler loading and morphology on the mechanical properties of contemporary composites. J Prosthet Dent, 87(6), 642649.

18. Koupis, N. S., Vercruysse, C. W., Marks, L. A., Martens, L. C., \& Verbeeck, R. M. (2004). Curing depth of (polyacidmodified) composite resins determined by scraping and a penetrometer. Dent. Mater., 20(10), 908-914.

19. Lee, H.-M., Kim, S.-C., Kang, K.-H., \& Chang, N.-Y. (2016). Comparison of the bonding strengths of second-and thirdgeneration light-emitting diode light-curing units. J Orthod, 46(6), 364-371.

20. Li, Y., Swartz, M., Phillips, R., Moore, B., \& Roberts, T. (1985). Materials science effect of filler content and size on properties of composites. J. Dent. Res, 64(12), 1396-1403.

21. Maruo, Y., Nishigawa, G., Irie, M., Yoshihara, K., \& Minagi, S. (2015). Flexural properties of polyethylene, glass and carbon fiber-reinforced resin composites for prosthetic frameworks. Acta Odontol. Scand, 73(8), 581-587.

22. Monaco, C., Bortolotto Ibarra, T., Arena, A., \& Krejci, I. (2015). Restoring nonvital premolars with composite resin onlays: effect of different fiber-reinforced composite layers on marginal adaptation and fracture load. $J$ Adhes Dent, 17(6), 567-574.

23. Nascimento, A. S., Lima, D. B., Fook, M. V. L., Albuquerque, M. S. D., Lima, E. A. d., Sabino, M. A., Physicomechanical characterization and biological evaluation of bulk-fill composite resin. Braz. Oral Res, 32.

24. Passos, S. P., Freitas, A. P., Jumaily, S., Santos, M. J. M., Rizkalla, A. S., \& Santos Jr, G. C. (2013). Comparison of mechanical properties of five commercial dental core build-up materials. Compendium, 34(1).

25. Price, R. B., Fahey, J., \& Felix, C. M. (2010). Knoop microhardness mapping used to compare the efficacy of LED, QTH and PAC curing lights. Oper Dent, 35(1), 58-68.

26. Rueggeberg, F., Caughman, W. F., \& Curtis, J. (1994). Effect of light intensity and exposure duration on cure of resin composite. Oper Dent, 19(1), 26-32.

27. Soh, M., Yap, A., \& Siow, K. (2003). Effectiveness of composite cure associated with different curing modes of LED lights. Oper Dent, 28(4), 371-377.

28. Spranley, T. J., Winkler, M., Dagate, J., Oncale, D., \& Strother, E. (2012). Curing light burns. Gen. Dent, 60(4), e210-214.

29. Sunnegårdh-Grönberg, K., Peutzfeldt, A., \& van Dijken, J. W. (2003). Flexural strength and modulus of a novel ceramic restorative cement intended for posterior restorations as determined by a three-point bending test. Acta Odontol. Scand, 61(2), 87-92.

30. Tolosa, M. C. C. G., Paulillo, L. A. M. S., Giannini, M., Santos, A. J. S. d., \& Dias, C. T. d. S. (2005). Influence of composite restorative materials and light-curing units on diametrical tensile strength. Braz. Oral Res, 19(2), 123-126.

31. Tyler, J., \& Poole, D. (1984). Uptake of fluoride by human surface enamel from ammonium bifluoride and consequent reduction in the penetration in vitro by caries-like lesions. Arch. Oral Biol, 29(12), 971-974.

32. Van Dijken, J. W., \& Sunnegårdh-Grönberg, K. (2006). Fiberreinforced packable resin composites in Class II cavities. $J$ Dent, 34(10), 763-769.

33. Vandewalker, J. P., Casey, J. A., Lincoln, T. A., \& Vandewalle, K. S. (2016). Properties of dual-cure, bulk-fill composite resin restorative materials. Gen. Dent, 64(2), 68.

34. Wang, L., D’Alpino, P. H. P., Lopes, L. G., \& Pereira, J. C. (2003). Mechanical properties of dental restorative materials: relative contribution of laboratory tests. J. Appl. Oral Sci, 11(3), 162-167.

35. Willems, G., Lambrechts, P., Braem, M., \& Vanherle, G. (1993). Composite resins in the 21st century. Quintessence Int, 24(9).

36. Xu, H. (2000). Whisker-reinforced heat-cured dental resin composites: effects of filler level and heat-cure temperature and time. J. Dent. Res, 79(6), 1392-1397.

37. Xu, H. H., Quinn, J. B., Smith, D. T., Giuseppetti, A. A., \& Eichmiller, F. C. (2003). Effects of different whiskers on the reinforcement of dental resin composites. Dent. Mater., 19(5), 359-367. 